\title{
Cluster based Routing Protocols for Wireless Sensor Networks: An Overview
}

\author{
Muhammad Nadeem Akhtar ${ }^{1}$, Arshad Ali ${ }^{2},{\text { Zulfiqar } \mathrm{Ali}^{3}, \text { Muhammad Adnan Hashmi }}^{4}$ and Muhammad Atif ${ }^{5}$ \\ Department of Computer Science \& Information Technology \\ The University of Lahore, Lahore, 55150, Pakistan
}

\begin{abstract}
Energy consumption of nodes in Wireless Sensor Networks (WSNs) is a very critical issue, particularly in scenarios where the energy of nodes cannot be recharged. Optimal routing approaches play a key role in energy utilization, so there is great importance of energy efficient routing protocols in WSNs. Energy efficient routing protocols in WSNs are categorized into four schemes, namely (i) communication model, (ii) topology based model, (iii) reliable routing, and (iv) network structure. Network structure category is further divided into flat and cluster-based approaches. This work focuses on a subtype of "network structure" scheme known as clustered based routing protocols, which are mainly used in WSNs for reduction in energy consumption. This work reviews and provides an overview of prominent cluster based energy efficient routing protocols on the basiss of some primary performance metrics such as (i) energy efficiency, (ii) algorithm complexity, (iii) scalability, (iv) data delivery delay, and (v) clustering approach. Finally, this work discusses some latest research trends with respect to cluster based energy efficient routing protocols in WSNs.
\end{abstract}

Keywords-Wireless sensor networks; network structure; clustering protocols; energy efficiency

\section{INTRODUCTION}

Since the last twenty years, there is a rapid growth with respect to technologies in the field of data communication networks. This technological progress facilitates organizations by providing very easy and secure working environment. Wireless networks enable organizations to get rid of expensive procedure of using cables for the purpose of connecting equipment located at different locations. This motivates organizations to use wireless networks for communication purpose.

From topological perspective, wireless networks are commonly classified into two modes i.e., (i) infrastructure mode, and (ii) ad hoc mode. The former supports communication between nodes through a Base Station (BS), while in ad hoc mode, all nodes can communicate with each other directly without requiring any infrastructure and no node is superior to any other node in the absence of any central entity.

A Wireless Sensor Networks (WSN) is a set of low-cost and small-sized sensor nodes having limited communication range, energy, processing, and storage capacity. From network design perspective, WSNs are classifies into structured and unstructured networks. In the former, the deployment of nodes is made with proper planning while in latter the same is done in an ad hoc manner [1].

A wireless sensor network is a combination of various sensor nodes connected with each other. Physical location where these nodes are deployed is known as a sensor field. Data from any node is transferred to other linked nodes and aggregated at sink node in order to be accessible to end users as shown in Fig.1 [2].

Each sensor node has four major hardware components i.e., (i) sensing unit, (ii) a processing unit, (iii) transmitting / receiving unit, and (iv) power unit. Each sensor comprises of application dependent two additional components, namely (i) location finder system, and (ii) mobilizer. Sensor and analog to digital converter (ADC) are two sub-parts of sensing unit. Initially, the data is observed by a sensor which is forwarded to ADC for conversion into digital form. Then, digital data is sent to the processing unit, which is usually linked with a storage unit consisting of a small storage capacity. In order to perform assigned activities, sensor units cooperate with each other by using procedures organized by processing unit. The transceiver helps a node in connecting with the network. Power unit, considered as the most important part, provides power to all the remaining units. The power may be provided through solar cells or by using power generator system (refer to Fig.2) [2].

Sensors used in WSNs have various kinds like acoustics, seismic visual, low sampling magnetic, thermal, radar and infrared. These sensors are capable of monitoring several conditions such as noise level, soil makeup, lightening, vehicular movement, temperature, humidity and pressure etc.

WSNs have various application areas i.e., performance monitoring of industrial machines, environmental monitoring, monitoring of health and military battlefield [3].

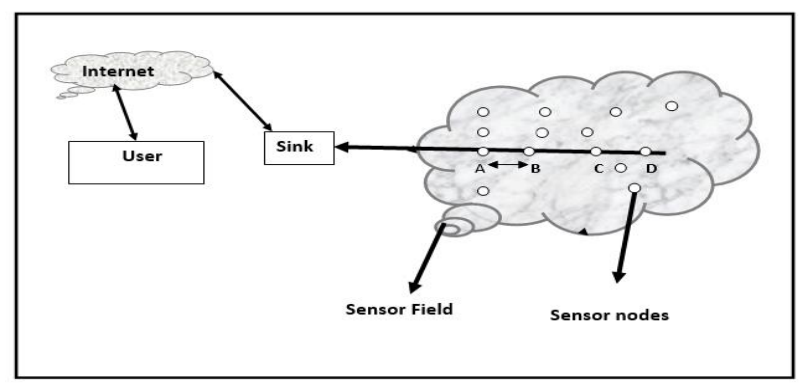

Fig. 1. Sensor Nodes in a Sensor Field. 


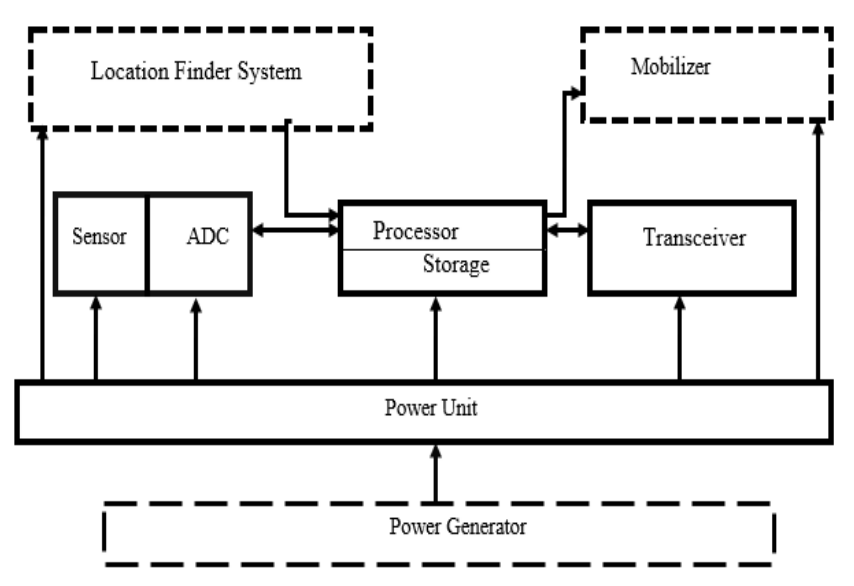

Fig. 2. Sensor Nodes' Hardware Components.

Routing protocols are of great attention because of versatilities lying in network architecture as well as in applications using wireless sensor networks. Traditional routing protocols are not applicable in WSNs; therefore WSNs require routing protocols different than traditional ones. Consequently, many energy-efficient routing protocols were developed for WSNs for providing efficient delivery of to the destination. Keeping in view the nature of application and network architecture, each energy-efficient routing protocol may possess specific features.

This research work provides an overview of existing energy-efficient cluster based routing protocols in the context of WSNs. Moreover, it lists a brief comparison of the studied protocols. The rest of the paper is organized as follows: Section II describes the related work. Section III provides the review of the cluster based hierarchical routing protocols of WSNs. Section IV gives comparative analysis of prominent hierarchical clustering protocols in terms of performance metrics and final Section V concludes the study.

\section{RELATED WORK}

Many surveys have already been conducted in the area of WSNs energy efficient routing protocols, from different perspectives. However, this paper reviews hierarchal / clusterbased routing protocols in WSNs.

In 2004, Al karaka and A E Kamal [4] surveyed routing techniques in WSNs. This work provided a taxonomy about WSN routing protocols by dividing them into two major categories (i) network structure and (ii ) protocol operation. The network structure is further divided into flat, hierarchal and location-based routing. The location based is further divided into (i) negation based, (ii) multipath based, (iii) query based, (iv) QoS based, and (v) coherent based routing. This work also exposed some design tradeoff between communication overheads and energy saving in some routing protocols paradigm.

In a survey in 2005, Kemal Akkaya et al. [5] classified WSNs routing protocols as data-centric, hierarchal and location-based. Each protocol was placed in one basic category, while a few protocols belonged to more than one class conserving various metrics such as QoS, network flow, and data aggregation. All of the clustering protocols were not discussed in this work.

In 2007, Abbasi et al. [6] presented a taxonomy of different clustering schemes and provided an overview of clustering protocols and algorithms from the perspective of variable convergence time and constant convergence time. Moreover, their study provided a comparison of some popular clustering methods.

In 2008, Deosarkar et al. [7] discussed cluster head $(\mathrm{CH})$ selection techniques on the basis of classification as (i) deterministic, (ii) adaptive, and (iii) combined metric schemes. The authors compared the cost of $\mathrm{CH}$ selection from various angles like cluster information, creation, and distribution of clusters.

In 2010, Shio Kumar Singh et al. [8] described cluster based energy efficient routing protocols in WSN. The authors highlighted some pitfall and disadvantages of individual protocols along with some future trends and constraints lying in this area.

In 2012, Xuxun Liu [9] comparatively expressed a better survey on cluster-based energy efficient WSN routing protocols. The author developed a novel taxonomy about clustering methods on WSN rather than detailed clustering attributes. This work analyzed some prominent clustering routing protocols in WSNs and compared them through different approaches as discussed in the taxonomy about the cluster (refer to Fig.3). The author described three clustering approaches i.e., (i) centralize, (ii) distributed, and (iii) hybrid. Centralize clustering approach is responsible for making clusters and $\mathrm{CH}$ selection. Distributed approach allows all cluster nodes to work as $\mathrm{CH}$ for the current round. Hybrid approach combines the properties of both centralize and distributed approaches.

In 2013, Nikolaos A. P et al. [10] presented a detailed survey on overall energy efficient WSN routing protocols by dividing them into four main categories on the basis of energy efficiency, nemly (i) network structure, (ii) communication model based, (iii) topology based, and (iv) reliable routing based. The first scheme is further divided flat and cluster based approaches. The second scheme is classified in three subtypes i.e., (i) query based, (ii) non query based/negation based, and (iii) coherent based. The third scheme is further divided into location-based and mobile agent based ones. The fourth scheme is divided into QoS based and multipath based schemes. Fig. 4 presents the complete picture of their division. The present work focuses cluster based routing protocols in detail.

In 2014, Agam Gupta and Anand Nayyar [11] discussed many routing protocols. Traditional routing protocols being used in WSNs lack in load balancing and efficiency of energy. The use of clustering not only improves network life time but also supports load balancing. There are many clusters and each cluster consists of many inter-connected sensor nodes, while one of them works as a cluster head $(\mathrm{CH})$. Each cluster head gathers data from the nodes belonging to the cluster and transfer that data to the BS (refer to Fig.5). There is intra cluster as well as inter cluster data communication between cluster head and member nodes of the cluster [11]. 


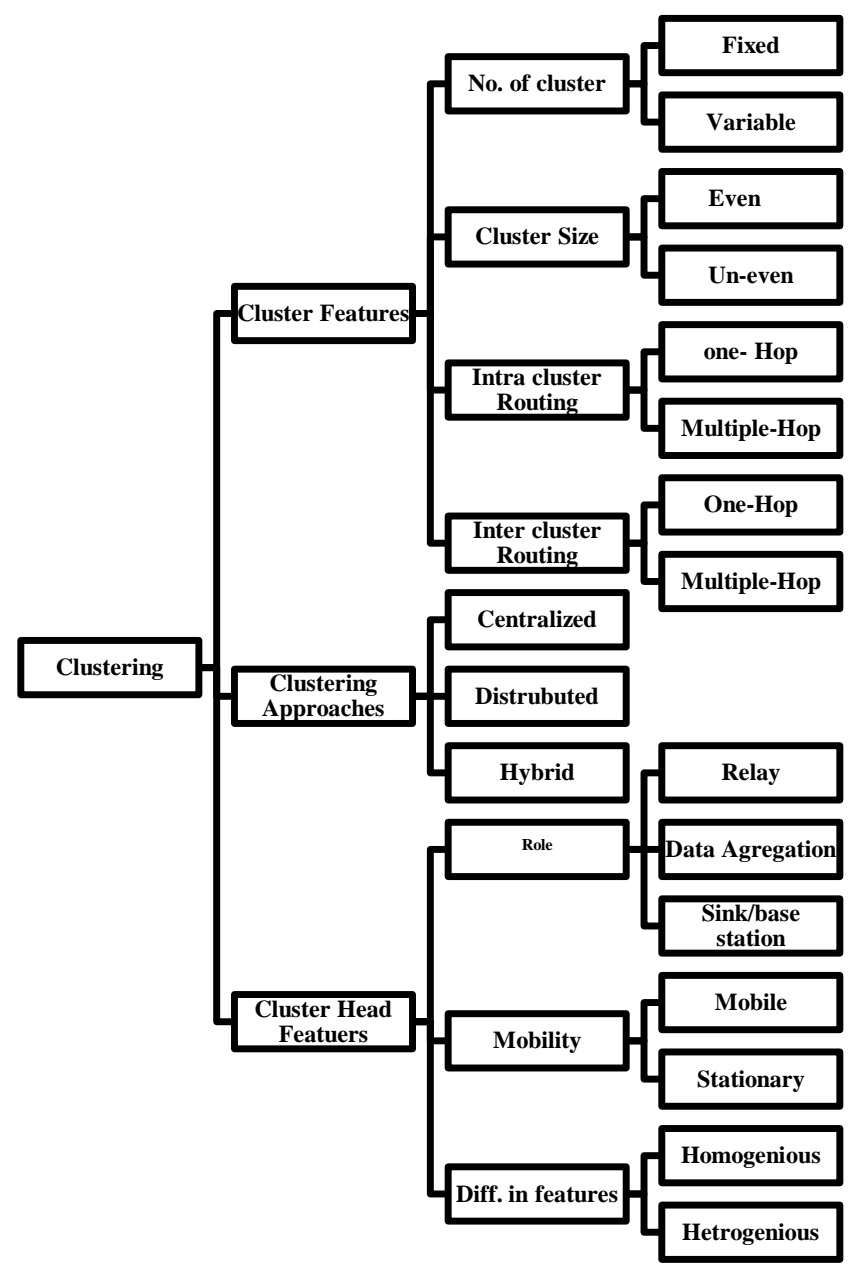

Fig. 3. Different Aspects of Cluster in WSNs [9].

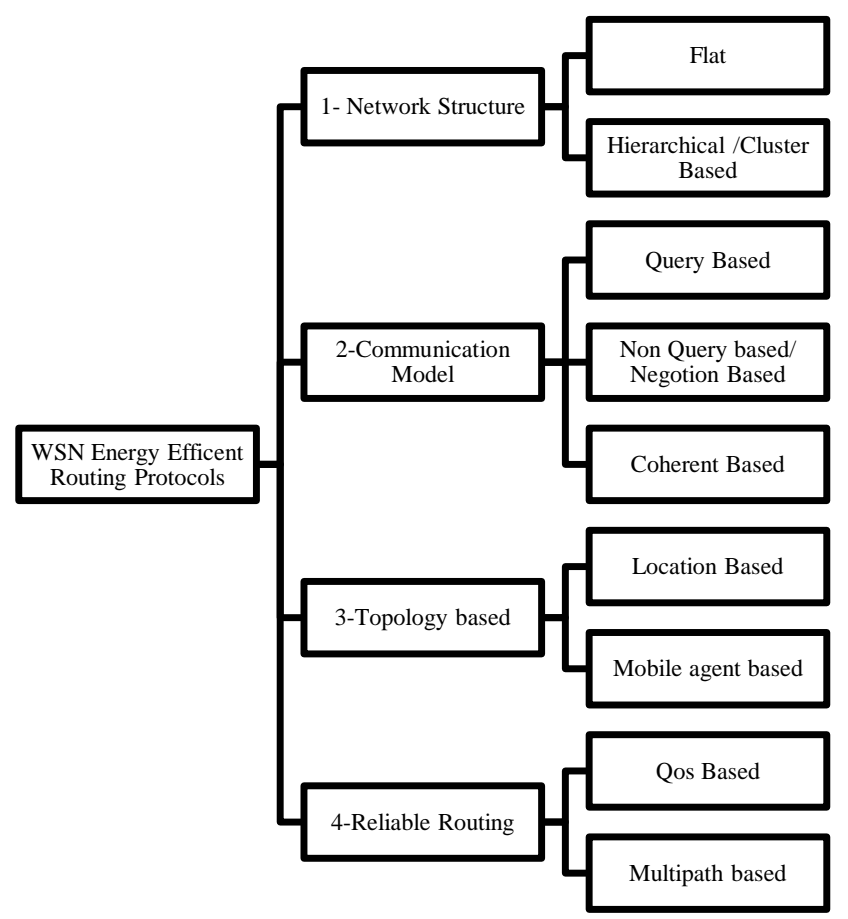

Fig. 4. Energy Efficient Routing Protocols in WSNs [10].

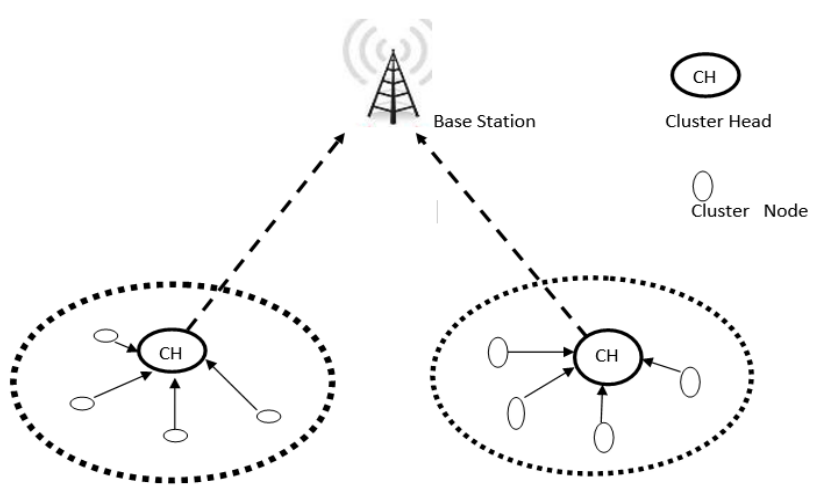

Fig. 5. Cluster in WSNs [11].

In 2015, Santar Paul Singh and SC Sharma [12] conducted a survey on cluster based energy efficient WSN routing protocols. The authors described taxonomy of WSN routing protocols into five categories, namely (i) initiator of communication, (ii) Path establishment, (iii) Network structure, (iv) protocol operation, and (v) next hope selection. Network structure scheme is Specifically further classified into (i) flat, (ii) cluster based, and (iii) location based. The authors in this survey did not review clustering protocols individually. They classified cluster-based routing protocols into three sub-categories, i.e., (i) block cluster-based, (ii)grid cluster based, and (c) chain cluster based. According to this classification, different clustering protocols lie under these three clustering schemes. In the end, the authors discussed some merits and limitations of some prominent cluster-based routing protocols.

In 2015, Priyanka Sharma and Inderjeet kaur [13] discussed WSNs routing protocols by classifying them into three main categories, i.e., (i) path establishment, (ii) network structure, and (c) protocol operation. First scheme path establishment is further divided into proactive, reactive and hybrid. Second scheme network structure is further divided into flat, hierarchal and location based. The third scheme is further classified into eight sub-types, namely (i) query, (ii) bio-Inspired, (iii) multipath, (iv) negation based, (v) QoS, (vi) non-coherent, (vii) coherent, and (viii) mobility. The authors discussed some metrics, pros, cons, and applications of some clustering protocols lying in above-mentioned categories.

In 2015, Ibrihich Ouafaa et al. [14] discussed and compared some prominent cluster-based routing protocols by classifying them into WSN and ad-hoc categories. The authors also compared these prominent protocols considering some important metrics.

In 2016, Yan et al. [15] classified WSNs routing protocols into data-centric, location-based and hierarchal depending on network structure. In data-centric approach, metadata approach is used by the protocols to sense and transmit information to base station. Hierarchal approach adopts clustering technique which can be made by grouping sensor nodes. The cluster reduces the energy utilization of sensor nodes. Clustering technique is more scalable and is used in a number of various applications. The location-based approach uses position/ location of nodes to route the data intelligently. 
In 2017, Syed Bilal Hussain Shah et al. [16] conducted a survey on hierarchal routing protocols in WSNs to increase network lifetime and conserve energy. In this survey, they reviewed some of the hierarchal routing protocols like LEACH, LEACH- TLCH, APTEEN, TEEN and proposed new scheme adaptive threshold. They also discussed some limitations of LEACH and some merits of newly proposed adaptive threshold. Adoptive Threshold attained good results as compared to some of the previously discussed schemes. But the authors did not studied or compared all hierarchal/ clustering protocols with the newly proposed scheme.

In 2018, G. Beni and C. Selden Christopher [17] discussed a few cluster-based protocols like LEACH, PEGASIS, HEED, TEEN and APTEEN from the perspective of comparing performance metrics like energy efficiency, cluster stability, delivery delay, and scalability. But the authors did not present a detailed survey on all hierarchal / cluster-based protocols of WSN.

Our work, to the best of our knowledge, is a more comprehensive study covering maximum number of famous hierarchal /cluster based protocols of WSNs with different clustering approaches.

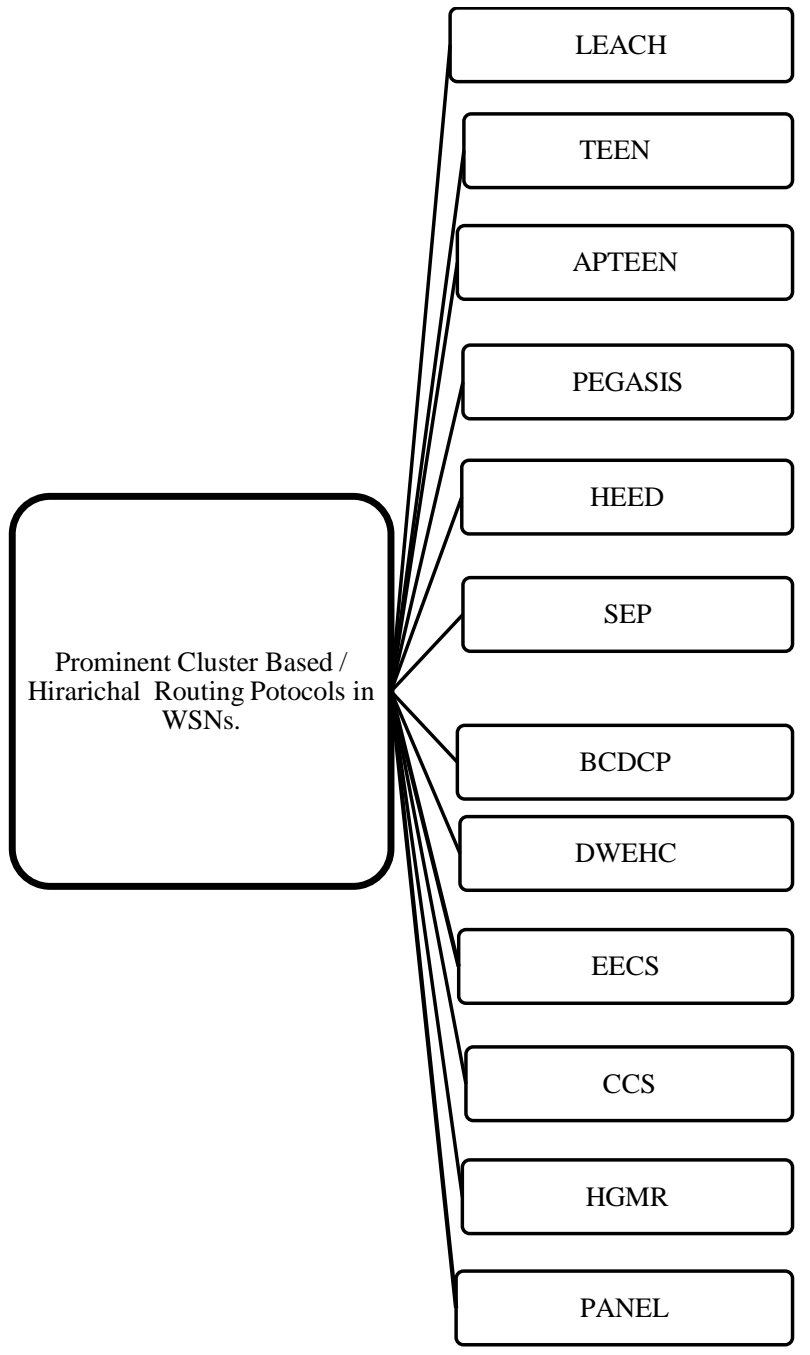

Fig. 6. Cluster-Based Routing Protocols in WSNs.

\section{Cluster-BASEd Routing Protocols IN WSNS}

This section provides an overview of prominent energy efficient cluster-based hierarchal routing protocols of WSNs. In cluster-based routing protocols, multiple nodes connected with each other in a sensor field make a group having one node among them as a cluster head. Data transformation from that particular cluster to sink node occurs through the cluster head $(\mathrm{CH})$. In this way, the energy of other nodes is saved. So in this classification of protocols, the major aspect is clustering. Fig. 6 depicts prominent cluster based / hierarchal routing protocols in WSNs while details of these protocols are provided in subsequent sub-sections.

\section{A. $\mathrm{LEACH}$}

In 2000, Heinzelman et al. [18] proposed one of the famous cluster based routing protocol for WSNs namely "Low Energy Adaptive Clustering Hierarchy" (LEACH). LEACH evenly distributed the load of energy between all sensors of the network using random based rotation of cluster head. In order to make dynamic networks more scalable and robust, LEACH used localize coordination. In LEACH, sensor nodes scattered in field organize themselves to make local clusters, among these sensor nodes one node becomes local base station or cluster head $(\mathrm{CH})$. This $\mathrm{CH}$ works as router, transfers the signals from all sensor nodes to the sink. LEACH saves energy due to transfer of data by $\mathrm{CH}$ rather than transferring of the data individually by all sensor nodes. Optimal number of nodes considered as cluster head are about five percent of the total nodes. In LEACH, all data processing including "data aggregation" and "data fusion" is held locally in the cluster which results in reduction of energy dissipation as well as increasing life time of the system. Cluster head is changed randomly, so energy dissipation between all nodes becomes balanced. $\mathrm{CH}$ changing decision is made by randomly selecting a number between 0 and 1 .

If selected number (shown in Eq.1) is below the threshold, node may become the $\mathrm{CH}$ for some specific round.

$T(n)= \begin{cases}\frac{P}{1-P\left(\operatorname{rmod} \frac{1}{p}\right)} & \text { if } n E G \\ 0 & \text { otherwise }\end{cases}$

In Eq.1, variable "P" is desired percentage of the $\mathrm{CH}$ ( for example .05), variable " $r$ " means current round whereas variable " $G$ " means a set of those nodes which are not selected as $\mathrm{CH}$ from last $1 / \mathrm{P}$ rounds.

The authors claimed that $\mathrm{LEACH}$ reduced 8 times energy as compared to direct communication (DC) and minimum transmission energy (MTE) protocol. LEACH, being a single hop routing protocol, possesses some deployment limits in larger networks where every individual node directly communicates to cluster head and sink.

\section{B. TEEN}

In 2001, Arati Manjeshwar and Dharma P. Agarwal [19] proposed "Threshold Sensitive Energy Efficient Sensor Network" (TEEN). According to authors TEEN was the first protocols of its time developed for "Reactive Networks". In reactive networks nodes immediately react to drastic and sudden changes in value of sensed attributes. 
In this protocol the $\mathrm{CH}$ broadcasts to its member nodes, at change time of every cluster, in addition to its attributes. TEEN was a combination of data-centric and hierarchical approach. Number of transmissions from member sensor nodes to $\mathrm{CH}$ are reduced in this protocol.

Two kinds of threshold values to ordinary member sensor nodes work in TEEN, i.e., (i) when a cluster is formed, and (ii) when $\mathrm{CH}$ broadcasts. The first value is called hard threshold (Ht) and second is called soft threshold ( $\mathrm{St}$ ).

Hard threshold (Ht) is sensed attribute's absolute/ minimum value, at which sensing node should turn on its transmitter for reporting to its $\mathrm{CH}$. Soft threshold (St) is small change occurred in sensed attributes, which causes to dictate the node to switch on the transmitter for the transmission purpose.

TEEN saves large amount of energy by reducing number of transmissions between cluster head and member sensor nodes.

The main shortcoming of TEEN is detection of dead nodes. Another limitation of TEEN is that it is difficult to forecast the reason if node is not sending the data. This can be happened because of two main reasons (i) node is unable to meet threshold value, and (ii) node may be dead. TEEN is suitable for time critical applications and suitable for energy consumption and response time [19].

\section{APTEEN}

In 2002, Arati Manjeshwar and Dharma P. Agarwal [20] proposed an improvement to overcome deficiencies of TEEN named as "Adaptive Periodic Threshold Sensitive Energy Efficient Sensor Network" (APTEEN). LEACH was considered suitable for proactive networks and TEEN was suitable for reactive networks. In APTEEN, the authors used hybrid network approach which had combined best features of both LEACH and TEEN. APTEEN was suitable for time critical events as well as to obtain data periodically. Simulation results showed that network lifetime and energy dissipation of APTEEN existed between TEEN and LEACH.

\section{PEGASIS}

In 2002, Stephanie Lindsey et al. [21] proposed a chain based protocol namely "Power-Efficient Gathering in Sensor Information Systems" (PEGASIS) which was an improvement over LEACH. In PEGASIS each node sends and receives data to only nearby neighbor nodes. Data reaches to the base station in turns, due to which energy consumption per round is reduced. All member sensor nodes are connected with each other in such a way that they make a chain. Using greedy algorithm, chain computation may be initialized by broadcasting data from a node or base station to all member sensor nodes, as shown in Fig. 7.

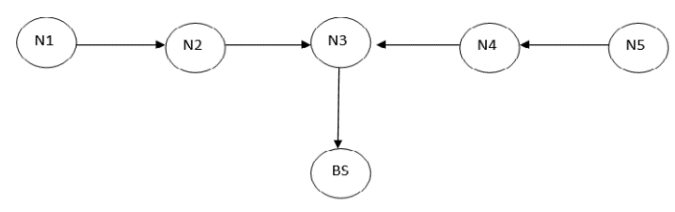

Fig. 7. Chaining in PEGASIS.

\section{E. HEED}

In 2004, Ossama Younis et al. [22] proposed protocol named as Hybrid, Energy-Efficient Distributed Clustering (HEED) which was very excellent clustering based protocol.

In HEED node's residual energy is parameter to elect the CH.Node's degree or density is used as a metric, in selection of a cluster to get power balancing. HEED was mainly an improvement over LEACH.

\section{F. SEP}

In 2004, G. Smaragdakis et al. [23] proposed a protocol named as Stable Election Protocol (SEP) for cluster-based Hetero-genius wireless sensor networks. SEP is an improved version of LEACH and works like it. SEP prolongs time interval known as "stability period" of the first node before its death, which is very crucial for such kind of applications where feedback from sensor network is considered very reliable. In SEP cluster head $(\mathrm{CH})$ is elected on the basis of energy as a "parameter". In this protocol a node independently selects itself as $\mathrm{CH}$ on the base of its own initial energy. SEP depends upon each node's weighted "election probability" to make $\mathrm{CH}$ with respect to each node's "remaining energy". From simulation results, it can be concluded that SEP had longer period stability and greater average throughput as compared to existing clustering based heterogeneous oblivious protocols. The study also showed that SEP is more resilient in advance node's energy efficiency.

\section{G. $B C D C P$}

In 2005, Siva D. Muruganathan et al. [24] proposed BaseStation Controlled Dynamic Clustering Protocol (BCDCP). BCDCP maintains clusters and routing paths by utilizing high energy base station. In this protocol cluster head rotations are performed randomly. Intensive energy tasks are performed in this protocol. In BCDCP main concept is formation of balance clusters where every cluster head serves an equal number of member sensor nodes by avoiding cluster head overload. In overall sensor field there is uniform placement of cluster heads. Data is transferred to the base station by utilizing $\mathrm{CH}$ to- $\mathrm{CH}$ routing.

\section{H. DWEHC}

In 2005, Ping Ding et al. [25] proposed a protocol namely Distributed Weight based Energy Efficient Clustering (DWEHC), in which every node in its enclosure region, first of all, locates its neighbor, calculates weight of itself that depends upon two factors namely, (i) its distance from the neighbor node and (ii) residual energy. In that enclosed region, a node having maximum weight is selected as a $\mathrm{CH}$. Other neighbor nodes become member node under this $\mathrm{CH}$ hierarchy. This clustering process finally terminates after seven iterations. This clustering process has no dependency on the size and topology of the network. The authors showed through simulation that this protocol performed well. The performance of this protocol was also analyzed from the perspective of Inter-cluster and intracluster communication. Finally, authors compared DWEHC performance with HEEDAMRP algorithm and concluded that it outperforms HEEDAMRP in "energy consumption" and better "cluster generation" perspectives. 


\section{I. $E E C S$}

In 2005 and 2006, Mao Ye et al. [26, 27] proposed Energy Efficient Clustering Scheme (EECS) for WSNs. EECS is a clustering algorithm that is more suitable for periodical data gathering applications. EECS is like a LEACH. In this scheme sensor network is divided into many clusters and data communication between $\mathrm{CH}$ and $\mathrm{BS}$ is single-hop. In this scheme, a node candidate to become a $\mathrm{CH}$ competes for a given round to gain the ability to elevate the $\mathrm{CH}$. During this competition, $\mathrm{CH}$ candidate nodes broadcast residual energy among their neighboring $\mathrm{CH}$ candidates. In this duration, if a node under consideration could not get another node with more residual energy than it, then itself becomes the $\mathrm{CH}$. Cluster formation of EECS is different from LEACH. EECS improves in the capability to LEACH by introducing dynamic size in clusters which are based on "distance" of the cluster from BS.

\section{J. CCS}

In 2007, Sung-Min Jung et al. [28] proposed a protocol as Concentric Clustering Scheme (CCS), to improve the performance of PEGASIS. As in PEGASIS, data transmission is redundant because when one node is selected as a head node regardless of the base station it takes data from both side nodes to convey it to BS, so data transmission from head node to $\mathrm{BS}$ become redundant. To cope with this issue of redundancy, CCS protocol was proposed. The main concept of CCS is to consider the location of the base station so that the lifetime and performance of WSNs can be prolonged. This became possible to achieve, using CCS. In CCS, WSNs are divided into concentric shaped clusters to give data transmission flow. CCS enhanced performance by using four processes, namely (i) level assignment to each node relevant to base station, (ii) constructing chain in level area using greedy algorithm, (iii) constructing chain between head nodes of each level, and (iv) transferring of data from higher level head node to other lower level head node. CCS saved $35 \%$ energy as compare to PEGASIS.

\section{K. $H G M R$}

In 2008, Dimitrios Koutsonikolas et al. [29] proposed a protocol named as Hierarchical geographic multicast routing (HGMR). HGMR is basically location-based and multicast protocols for WSNs. Impeccably it incorporates innovations in the "locations based" \& "multicast" and it optimizes them for WSNs. HGMR performs this by concurrently providing scalability and energy efficiency to the networks of large size. It can be concluded from simulation results that HGMR has combined the strength of two protocols namely Hierarchal Rendezvous Point Multicast (HRPM) and Geographic Multicast Routing (GMR). HGMR protocols decompose multicast groups into the subgroups. It uses GMR's local multicast scheme to forward the data packets with multi branches of a Multicast tree in the single transmission. In HGMR, using mobile Geographic Hashing mechanism, multicast groups can be divided into their subgroups. The deployed area is divided into partitions of various equal sized square shaped subdomains called "Cells" and each cell is consisted of subgroups of members having managable size. There exists an access point (AP) in each cell which is responsible for all members of that cell. A Rendezvous Point (RP) manages all the APs.

\section{PANEL}

In 2007 and 2010, Levente Buttyan and Peter Schaffer [30, 31] proposed a protocol named as Position-based Aggregator Node ELection (PANEL) for WSNs. There exist some other aggregator node election protocols but PANEL has a novelty from them in a sense that it supports such kind of sensor network applications which are asynchronous. The sensor collects the reading information through the base station after some delay. Main motivational factor in the design of PANEL was its support for reliable and consistent application of data storage like TinyPEDS. PANEL deals with load balancing and also supportx intra and intercluster routing by allowing communication between sensor and aggregator, aggregators itself, an aggregator to BS and BS to aggregators. Cluster formation and energy consumption capabilities of PANEL are better than HEED. Following are key merits of PANEL: (1) PANEL is energy efficient ensuring load balancing due to the election of each node as an aggregator (2) Beside synchronous scenes this protocol also supports the asynchronous application.

Following are key limitations of PANEL: (i) The supposition that cluster formation is found/ determined before deployment thus cannot be applied upon WSN dynamics, (ii) it has information about the geographical position of the nodes, that is used to find which node must be an aggregator. In WSNs there is a constraint that geographical position is not always available except some special conditions like hardware and software having GPS feature, and (iii) an assumption about PANEL described by the authors is that within cluster nodes form a subnetwork, due to this there may occur such a situation that nodes within the cluster could not hear the announcement of nodes closest to reference point, and they may elect aggregator to another node.

\section{COMPARATIVE ANALYSIS AND RESEARCH CHALlENGES}

Table 1 shows a comparative analysis of various clusterbased protocols (discussed earlier in Section III) on the basis of different performance metrics, namely (i) energy efficiency, (ii) algorithm complexity, (iii) delay in data delivery, (iv) scalability, and (v) clustering approach. A tradeoff was observed in terms of energy efficiency and data delivery delay, i.e., BCDCP is very poor regarding energy efficiency but offers small delay. It was also observed that some protocols perform much better in terms of scalability; however, their performance is lower if other metrics are taken into account i.e., scalability of HGMR is very high while having very poor energy efficiency. It is worth mentioning that almost all selected protocols in this review follow the distributed clustering approach. Algorithm complexity is noticed from very low to very high.

The following research challenges require attention from research community

- The design of energy efficient cluster based protocols for wireless body area networks for the purpose of improving overall energy efficiency is an interesting domain to explore. 
- Further investigation is required considering integration of these protocols with technologies such as "Internet of things", "Vehicular Ad hoc Networks" and many others.

- Sensor nodes are deployed on vehicles in order to monitor events. Data aggregation is an important issue in VANTEs keeping in view high mobility of vehicular nodes.

- Security is one of the main concerns in WSNs due to its operation in open environment which requires serious efforts. For secure data transmission, the existing security approaches cannot be applied in present form due to limited resources of WSNs. Thus, there is need of mechanisms which provide secure data transmission by using less energy resources.

- The design of routing protocols in the context of Internet of Things requires attention from research community, an overview of the same is provided in [32].

TABLE I. COMPARISON BETWEEN PROMINENT CLUSTERING BASED ROUTING PROTOCOLS IN WSNS

\begin{tabular}{|l|l|l|l|l|l|}
\hline $\begin{array}{l}\text { Prominent } \\
\text { Cluster } \\
\text { based } \\
\text { Routing } \\
\text { Protocols }\end{array}$ & $\begin{array}{l}\text { Energy } \\
\text { Efficiency }\end{array}$ & $\begin{array}{l}\text { Algorithm } \\
\text { Complexity }\end{array}$ & $\begin{array}{l}\text { Delay in } \\
\text { data } \\
\text { delivery }\end{array}$ & Scalability & $\begin{array}{l}\text { Clustering } \\
\text { Approach }\end{array}$ \\
\hline LEACH & Very Poor & Low & $\begin{array}{l}\text { Very } \\
\text { small }\end{array}$ & Very Low & Distributed \\
\hline TEEN & Very High & High & Small & Low & Distributed \\
\hline APTEEN & Medium & Very High & Small & Low & Distributed \\
\hline PEGASIS & Poor & High & $\begin{array}{l}\text { Very } \\
\text { Large }\end{array}$ & Very Low & Distributed \\
\hline HEED & Medium & Medium & Medium & Medium & Distributed \\
\hline SEP & Medium & Very Low & $\begin{array}{l}\text { Very } \\
\text { Small }\end{array}$ & Medium & Distributed \\
\hline BCDCP & Very Poor & Very High & Small & Very Low & Centerlize \\
\hline DWEHC & Very High & Medium & Medium & Medium & Distributed \\
\hline
\end{tabular}

\section{CONCLUSION}

Wireless sensor networks (WSNs) remained an emerging area of research for the last two decades. There are various applications of WSNs like industrial machine performance, environmental monitoring, health monitoring, and military battlefield. Major short fall of WSNs is energy dissipation of nodes deployed in the field of these application areas. Optimal and effective routing protocols and approaches play a key role in energy utilization, so the importance of energy efficient routing protocols in WSNs is significant. In this paper, we reviewed well-known cluster-based energy efficient routing protocols in WSNs. This work highlighted a few clustering approaches and characteristics considered in energy efficient routing protocols. On the basis of primary performance metrics i.e. energy efficiency, algorithm complexity, delay in data delivery, scalability and clustering approach, a comparative analysis has been done among prominent cluster based energy efficient routing protocols used in WSNs. This study concludes that there is not any single protocol which has the capability to perform excellently considering all metrics. If one protocol is good in energy dissipation, it may have more delivery delay or its algorithm may be complex, on the other hand if a protocol offers less delay or low complexity in algorithm then it may be less energy efficient.

\section{REFERENCES}

[1] Feeney, Laura Marie, and Martin Nilsson. "Investigating the energy consumption of a wireless network interface in an ad hoc networking environment." In INFOCOM 2001. Twentieth Annual Joint Conference of the IEEE Computer and Communications Societies. Proceedings. IEEE, vol. 3, pp. 1548-1557. IEEE, 2001.

[2] F. Akyildiz, W. Su, Y. Sankarasubramaniam, and E. Cayirci, "Wireless sensor networks: a survey," Computer networks, vol. 38, no. 4, pp. 393 422, 2002.

[3] Guoe, Menting, Jiadue Shan, and Yicheo Yong. "Evaluation of sensor network capability in a practical problem." INTERNATIONAL JOURNAL OF ADVANCED AND APPLIED SCIENCES 1, no. 7 (2014): 1-9.

[4] J. N. Al-Karaki, and A. E. Kamal, "Routing techniques in wireless sensor networks: a survey," IEEE wireless communications, vol. 11, no. 6, pp. 6-28, 2004.

[5] K. Akkaya, and M. Younis, "A survey on routing protocols for wireless sensor networks," Ad hoc networks, vol. 3, no. 3, pp. 325-349, 2005.

[6] A. A. Abbasi, and M. Younis, "A survey on clustering algorithms for wireless sensor networks," Computer Communications, vol. 30, no. 14, pp. 2826-2841, 2007.

[7] Deosarkar, Bhaskar P., Narendra Singh Yadav, and R. P. Yadav. "Clusterhead selection in clustering algorithms for wireless sensor networks: A survey." In Computing, Communication and Networking, 2008. ICCCn 2008. International Conference on, pp. 1-8. IEEE, 2008.

[8] S. K. Singh, M. Singh, and D. Singh, "A survey of energy-efficient hierarchical cluster-based routing in wireless sensor networks," International Journal of Advanced Networking and Application (IJANA), vol. 2, no. 02, pp. 570-580, 2010.

[9] X. Liu, "A survey on clustering routing protocols in wireless sensor networks," sensors, vol. 12, no. 8, pp. 11113-11153, 2012.

[10] N. A. Pantazis, S. A. Nikolidakis, and D. D. Vergados, "Energy-efficient routing protocols in wireless sensor networks: A survey," IEEE Communications surveys \& tutorials, vol. 15, no. 2, pp. 551-591, 2013.

[11] A. Nayyar, and A. Gupta, "A comprehensive review of cluster-based energy efficient routing protocols in wireless sensor networks," IJRCCT, vol. 3, no. 1, pp. 104-110, 2014.

[12] S. P. Singh, and S. Sharma, "A survey on cluster-based routing protocols in wireless sensor networks," Procedia computer science, vol. 45, pp. 687-695, 2015.

[13] P. Sharma, and I. Kaur, "A Comparative Study on Energy Efficient Routing Protocols in Wireless Sensor Networks," International Journal of Computer Science Issues (IJCSI), vol. 12, no. 4, pp. 98, 2015.

[14] Ouafaa, Ibrihich, Laassiri Jalal, Krit Salah-ddine, and El Hajji Said. "The comparison study of hierarchical routing protocols for ad-hoc and wireless sensor networks: A literature survey." In Proceedings of the 
The International Conference on Engineering \& MIS 2015, p. 32. ACM, 2015.

[15] J. Yan, M. Zhou, and Z. Ding, "Recent advances in energy-efficient routing protocols for wireless sensor networks: A review," IEEE Access, vol. 4, pp. 5673-5686, 2016.

[16] Shah, Syed Bilal Hussian, Yin Fuliang, Inam Ullah Khan, Chen Zhe, and Muhammad Zakarya. "Collating and Analysing State-of-the-Art Hierarchical Routing Protocols in WSN to Increase Network Lifetime and Conserve Energy." In Proceedings of the International Conference on Future Networks and Distributed Systems, p. 31. ACM, 2017.

[17] Beni, G., and C. Seldev Christopher. "Analysis of Energy Efficient Routing Protocols in Wireless Sensor Networks." vol 14 Taga journal, 2018.

[18] W. B. Heinzelman, A. P. Chandrakasan, and H. Balakrishnan, "An application-specific protocol architecture for wireless microsensor networks," IEEE Transactions on wireless communications, vol. 1, no. 4, pp. 660-670, 2002.

[19] Manjeshwar, Arati, and Dharma P. Agrawal. "TEEN: a routing protocol for enhanced efficiency in wireless sensor networks." In null, p. 30189a. IEEE, 2001.

[20] Manjeshwar, Arati, and Dharma P. Agrawal. "APTEEN: A hybrid protocol for efficient routing and comprehensive information retrieval in wireless sensor networks." In ipdps, p. 0195b. IEEE, 2002.

[21] Lindsey, Stephanie, and Cauligi S. Raghavendra. "PEGASIS: Powerefficient gathering in sensor information systems." In Aerospace conference proceedings, 2002. IEEE, vol. 3, pp. 3-3. IEEE, 2002.

[22] O. Younis, and S. Fahmy, "HEED: a hybrid, energy-efficient, distributed clustering approach for ad hoc sensor networks," IEEE Transactions on mobile computing, vol. 3, no. 4, pp. 366-379, 2004.

[23] G. Smaragdakis, I. Matta, and A. Bestavros, SEP: A stable election protocol for clustered heterogeneous wireless sensor networks, Boston University Computer Science Department, 2004.
[24] S. D. Muruganathan, D. C. Ma, R. I. Bhasin, and A. O. Fapojuwo, "A centralized energy-efficient routing protocol for wireless sensor networks," IEEE Communications Magazine, vol. 43, no. 3, pp. S8-13, 2005.

[25] P. Ding, J. Holliday, and A. Celik, "Distributed energy-efficient hierarchical clustering for wireless sensor networks," Distributed computing in sensor systems, pp. 466-467, 2005.

[26] Ye, Mao, Chengfa Li, Guihai Chen, and Jie Wu. "EECS: an energy efficient clustering scheme in wireless sensor networks." In Performance, Computing, and Communications Conference, 2005. IPCCC 2005. 24th IEEE International, pp. 535-540. IEEE, 2005.

[27] M. YE, C. LI, G. CHEN, and J. WU, "An Energy Efficient Clustering Scheme in Wireless Sensor Networks," Ad Hoc \& Sensor Wireless Networks, vol. 17, pp. 33, 2006.

[28] Jung, Sung-Min, Young-Ju Han, and Tai-Myoung Chung. "The concentric clustering scheme for efficient energy consumption in the PEGASIS." In Advanced Communication Technology, The 9th International Conference on, vol. 1, pp. 260-265. IEEE, 2007.

[29] D. Koutsonikolas, S. M. Das, Y. C. Hu, and I. Stojmenovic, "Hierarchical geographic multicast routing for wireless sensor networks," Wireless networks, vol. 16, no. 2, pp. 449-466, 2010.

[30] Buttyan, Leventa, and Peter Schaffer. "PANEL: Position-based Aggregator Node Election in Wireless Sensor Networks." In Mobile Adhoc and Sensor Systems, 2007. MASS 2007. IEEE International Conference on, pp. 1-9. IEEE, 2007.

[31] L. Buttyán, and P. Schaffer, "Position-based aggregator node election in wireless sensor networks," International Journal of Distributed Sensor Networks, vol. 6, no. 1, pp. 679205, 2010.

[32] Belhaj, Salem, and Sofian Hamad. "Routing protocols from wireless sensor networks to the internet of things: An overview." INTERNATIONAL JOURNAL OF ADVANCED AND APPLIED SCIENCES 5, no. 9 (2018): 47-63. 